\title{
Hypothalamic inflammation in humans is not reversed by a profound weight loss and an improved insulin sensitivity due to bariatric surgery
}

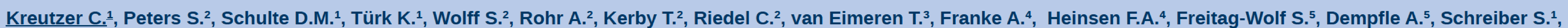
Laudes M. ${ }^{1}$

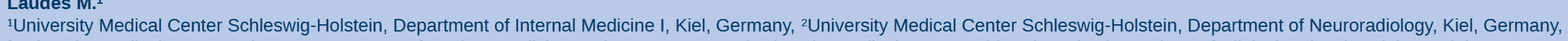

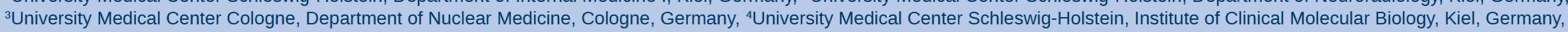
5University Medical Center Schleswig-Holstein, Department of Medical Informatics and Statistics, Kiel, Germany

\section{Introduction}

Obesity is associated with hypothalamic inflammation $(\mathrm{HI})$ in animal models. The mediobasal hypothalamus $(\mathrm{MBH})$ is a brain region crucial for body weight control as it regulates appetite, which leads to changes in nutritional intake. While MRI studies in obese human subjects also found an increased density in the $\mathrm{MBH}$, it remains unclear (1) if $\mathrm{HI}$ causes neuronal death and (2) if $\mathrm{HI}$ reverses during weight loss.

\section{Patients and methods}

$n=50$ obese subjects and $n=50$ age- and gender-matched controls where examined. MRI scans including spectroscopy were performed (s.Fig.1). Also, detailed nutritional questionnaires, serum lipidomics, 16s rDNA microbiome sequencing data as well as SNP genotyping data were obtained. $n=10$ obese subjects underwent bariatric surgery followed by a second MRI.

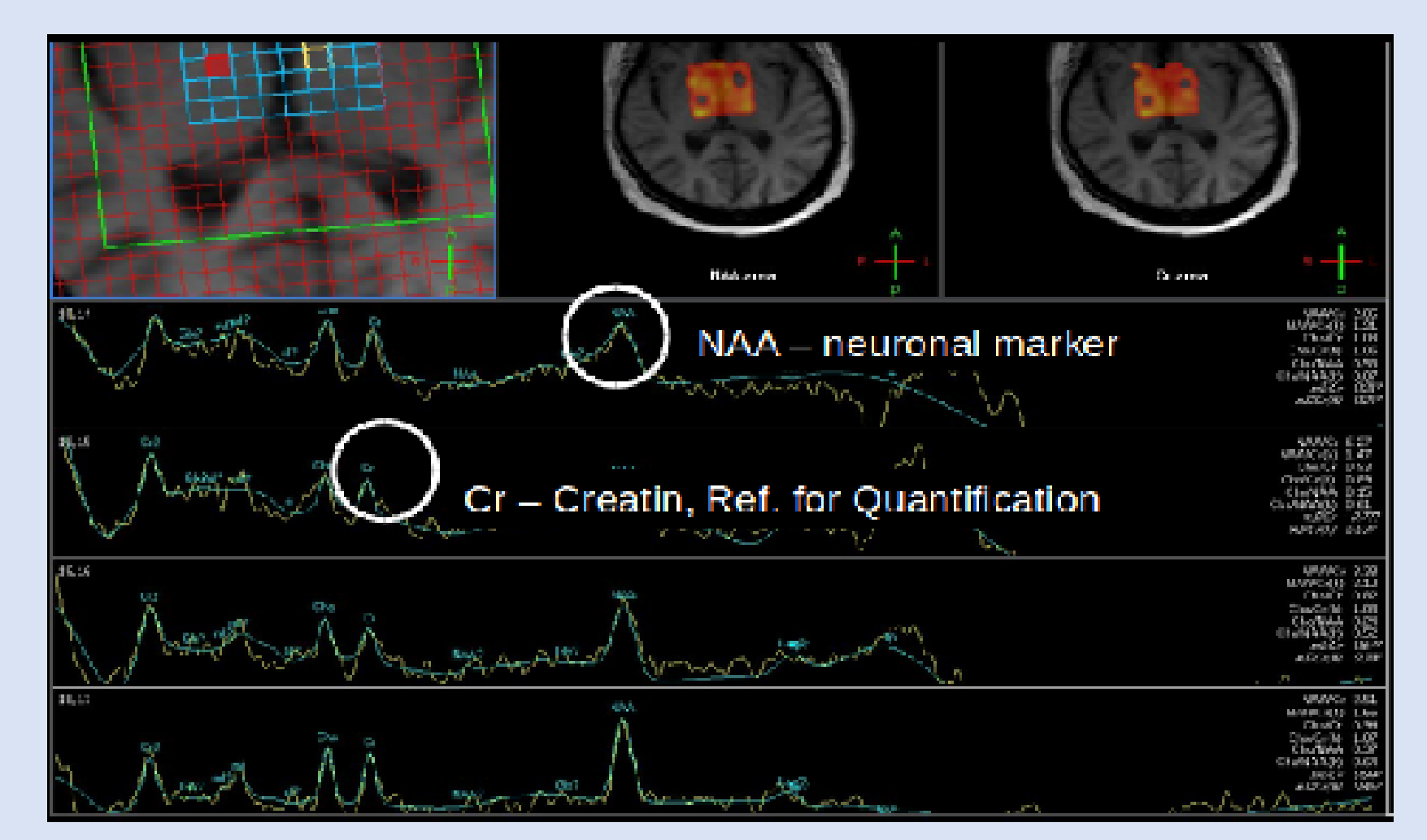

Fig.1: Spectroscopy illustration, method: ${ }^{1} \mathrm{H}$ NMR T2 relaxation time

$N$-Acetylaspartat (NAA) and Creatin (Cr)

measurements where used to calculate the NAA/Cr

ratio, a marker for neuronal cell count.

\section{Results}

1) Obese subjects exhibit an increased density in the left, but not the right $\mathrm{MBH}$ compared to non-obese controls.

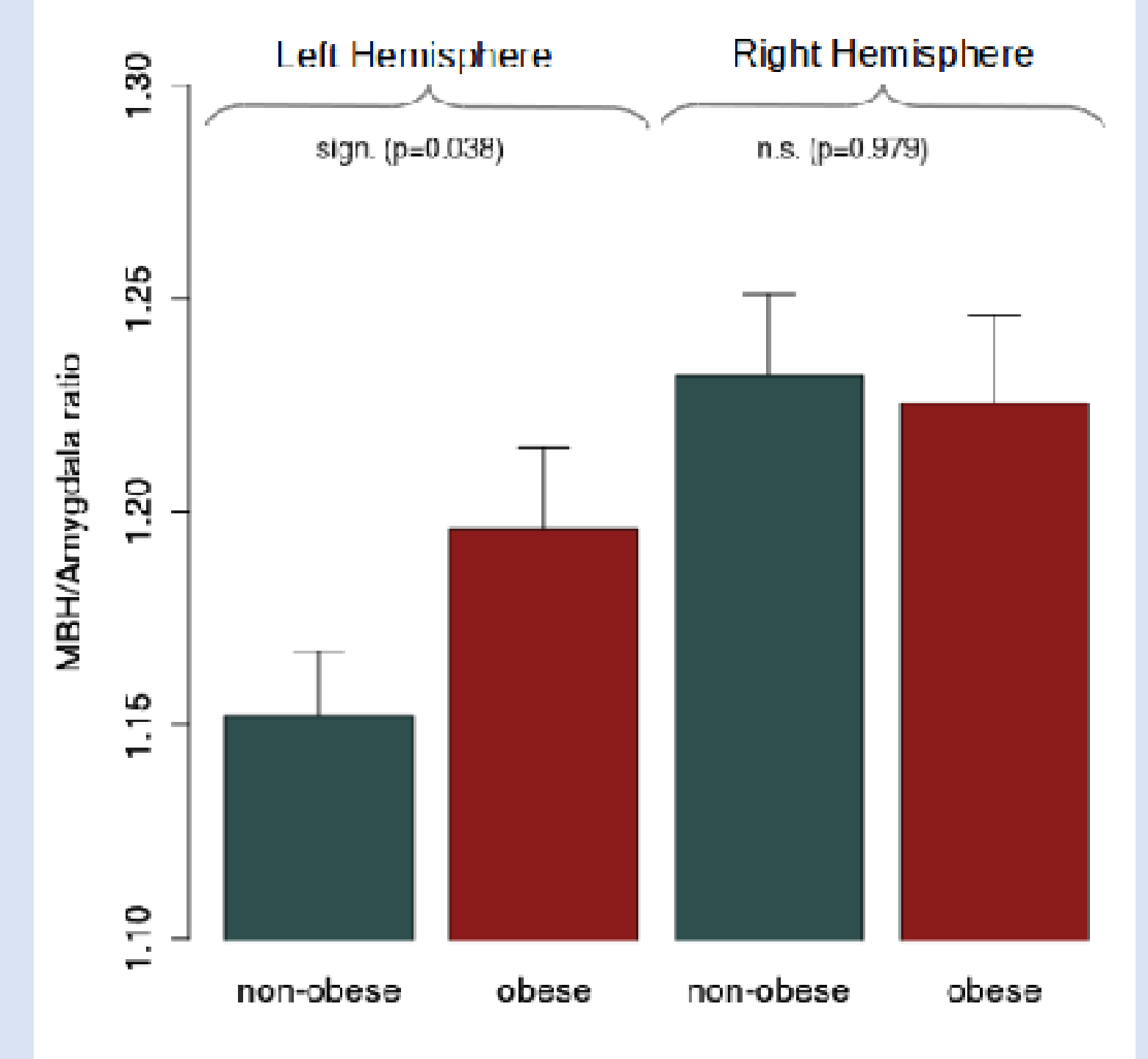

Fig.2: Comparison of MBH Density between nonobese and obese human subjects

The $\mathrm{MBH} /$ Amygdala ratio revealed significant differences (2-sided t-test) between the two obesity groups within the left hemisphere.

2) The number of neurons is not altered in the left $\mathrm{MBH}$ of obese human subjects despite increased MBH-Density, indicating that neurons might not be negatively affected by the inflammatory process.

善事

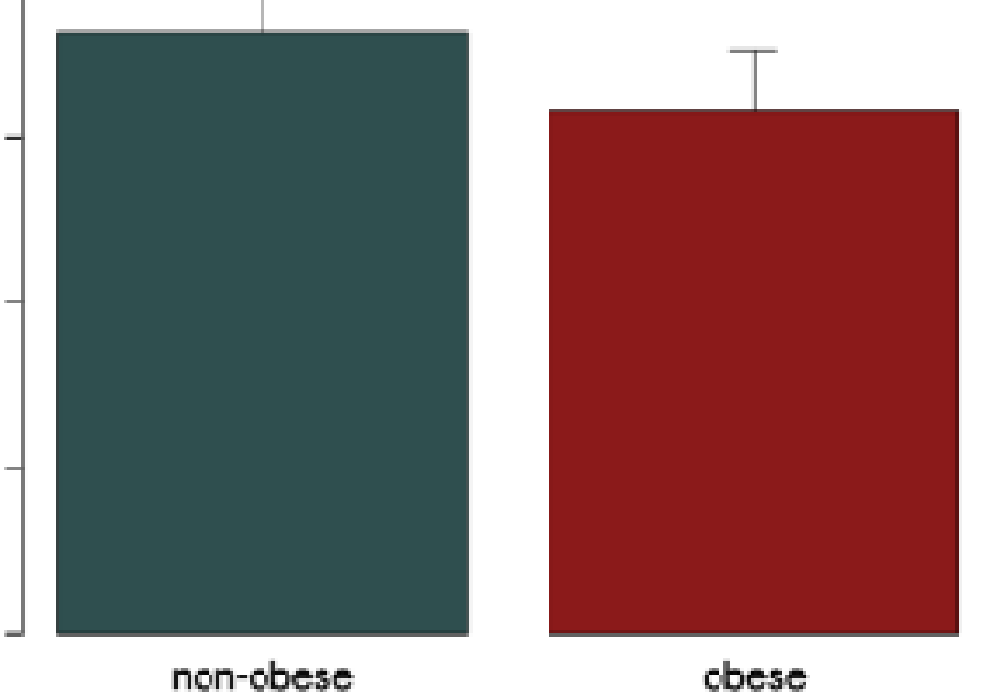

Fig.3: Comparison of neuronal cell count of non-obese vs. obese humans

The NAA/Cr ratio shows no significant differences (t-test) in cell numbers among the weight groups.

3) Weight loss due to bariatric surgery does not influence MBH-Density in obese humans.

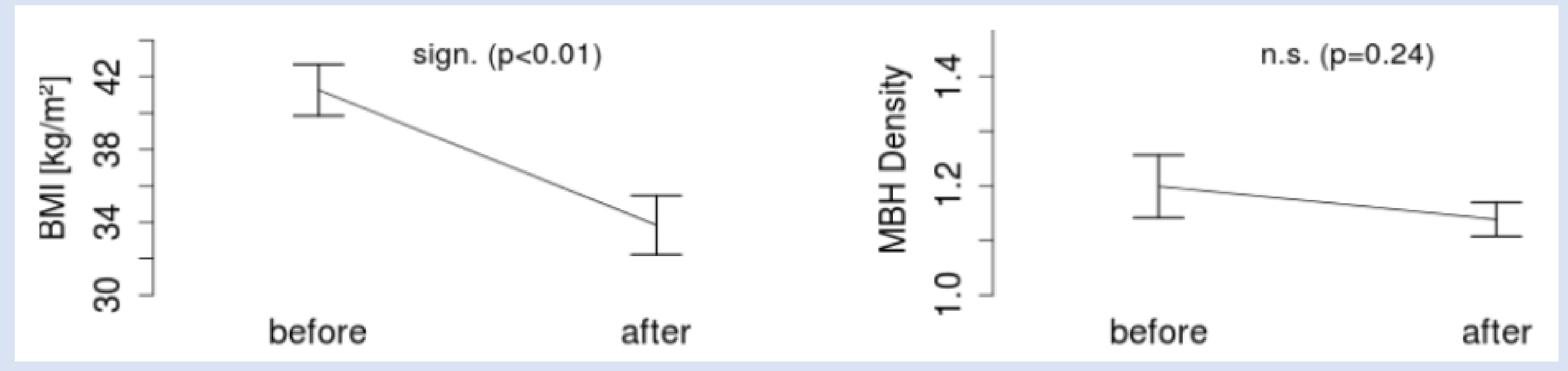

Fig.4: Before and after bariatric surgery

$\mathrm{BMI}$ and $\mathrm{MBH}$-Density, measured as MBH/Amygdala ratio. Before- and

after-comparison via t-test.
4) Increased density in the left $\mathrm{MBH}$ of obese humans is associated with systemic inflammation (A) but not with systemic glucose metabolism (B).

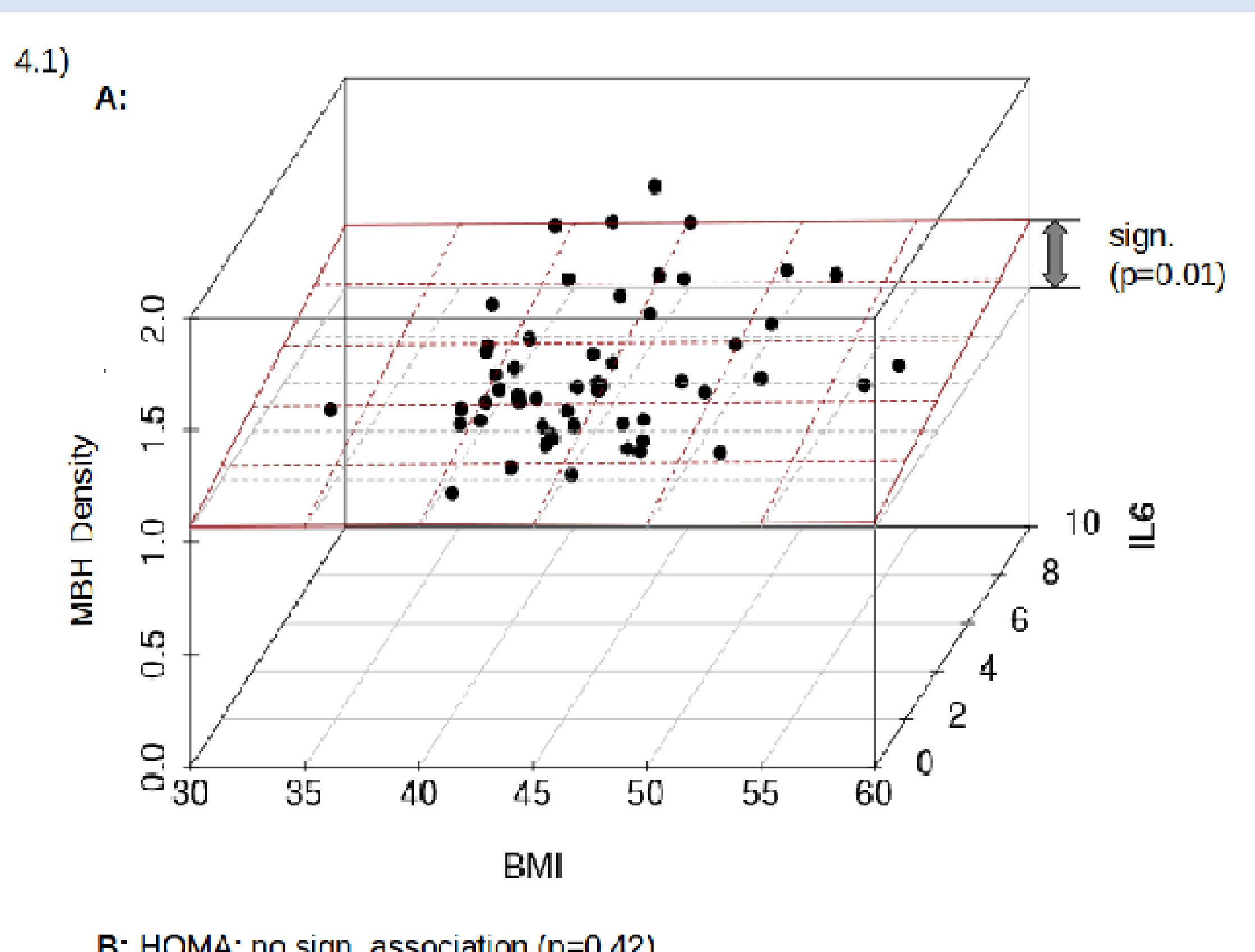

Fig.5: 3D-Scatterplot of MBH-Density, IL6 and BMI, within the obesity group

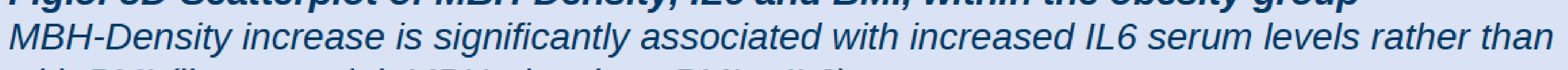
with $B M I$ (linear model: $M B H \_$density $\left.~ B M I+I L 6\right)$.

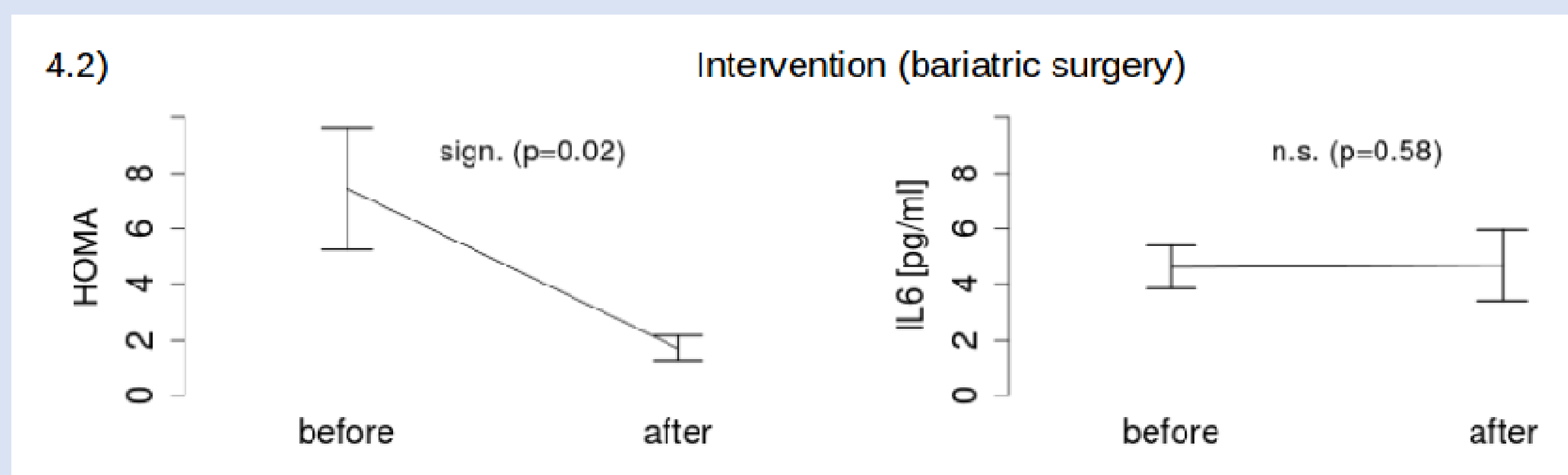

Fig.6: Before and after bariatric surgery

IL6 and HOMA (Homeostasis Model assessment - as a measure for insulin resistance). Before- and after-comparison via t-test.

5) GUT-BRAIN-AXIS: Alterations in the MBH-Density in obese humans are significantly associated with changes in the gut microbiome.

\begin{tabular}{|l|l|l|l|l|l|}
\hline Phylum & Order & Genus & p-value & rho & BMI correlation (p-value) \\
\hline Proteobacteria & Burkholderiales & Parasutterella & 0.0073 & -0.383 & n.s. (0.878) \\
\hline Bacteriodetes & Bacteriodales & Unclass. Marinilabiliaceae & 0.0398 & -0.299 & n.s. (0.539) \\
\hline
\end{tabular}

Table 1: Univariate correlation tests of the core measurable gut microbiome $(n=42)$ and $\mathrm{MBH}$-Density (left). The two bacterial genera are associated with $\mathrm{MBH}$-Density but not with BMI. Correlation method: Spearman.

6) A high-fat diet in obese humans induces a microbiome shift which is associated with the increase in the left MBH-Density.

\begin{tabular}{|l|l|l|}
\hline Nutrient & p-value & rho \\
\hline Fats & 0.044 & -0.30 \\
\hline Carbohydrates & n.s. (0.6) & -0.08 \\
\hline Proteins & n.s. (0.1) & -0.28 \\
\hline
\end{tabular}

Table 2: Univariate correlation tests of nutrients and Parasutterella sp. Data about nutritional intake where obtained by analysing food frequency questionnaires. Correlation method: Spearman.

\section{Discussion}

Obese human subjects exhibit an increased MRI-Density of the $\mathrm{MBH}$ suggesting $\mathrm{HI}$. While $\mathrm{HI}$ and IL6 are not reversed by a significant weight loss due to bariatric surgery, the finding that the number of neurons is not altered in the $\mathrm{MBH}$ by $\mathrm{HI}$ might suggest that the function of the $\mathrm{MBH}$ in terms of appetite regulation might be reversible.

Disturbances in the gut-brain-axis induced by an unhealthy diet might in part explain the obesity associated hypothalamic pathology. 